VOL. $50(1994)$ [513-518]

\title{
ON COMPLETELY PRINCIPALLY INJECTIVE RINGS
}

\author{
W.K. Nicholson and M.F. YousiF
}

\begin{abstract}
A ring $R$ is called right principally injective (right $P$-injective) if every $R$-linear map from a principal right ideal of $R$ can be extended to $R$. If every ring homomorphic image of $R$ is right $P$-injective, $R$ is called completely right $P$-injective (right $C P$-injective). In this paper we characterise completely quasi-Frobenius rings in terms of $C P$-injectivity.
\end{abstract}

A ring $R$ is called right principally injective (right $P$-injective) if every $R$-linear map $a R \rightarrow R, a \in R$, is given by left multiplication by an element of $R$, equivalently if $\ell[r(a)]=R a$ for all $a \in R$ where $\ell(x)$ and $r(x)$ denote the left and right annihilator of a set $x$, respectively. We studied these rings in [8]; and commutative $p$-injective rings are discussed by Camillo in [2].

A ring $R$ is called completely right $P$-injective (right $C P$-injective) if every ring image of $R$ is right $P$-injective. Left $P$-injective and left $C P$-injective rings are defined analogously. In general, the prefix "completely" signifies that the property in question holds in every ring image of $R$. The class of completely quasi-Frobenius rings has been studied in detail, see Faith [3]. In this paper we characterise these rings in terms of $P$-injectivity.

A module $M$ is uniserial if its submodules are linearly ordered by inclusion, and $M$ is serial if it is a finite direct sum of uniserial submodules. A ring $R$ is right (uniserial) serial if $R_{R}$ is a right (uniserial) serial module, with a similar definition on the left, and a serial ring is one that is both right and left serial. A commutative uniserial ring is called a valuation ring. A ring $R$ is called right GPF-ring (Generalised PseudoFrobenius) if $R$ is a semiperfect right $P$-injective ring with essential right socle. GPF rings were studied in great detail in [8].

Theorem 1. Suppose $R$ is a left perfect, right $C P$-injective ring. Then $R$ is left Artinian and left serial.

Proof: Let $A$ be a two-sided ideal of $R$. Then $\bar{R}=R / A$ is a right GPF-ring. By [8, Theorem 2.3], Soc $(\bar{R} \bar{R})$ is finitely generated and essential as a left ideal of $\bar{R}$.

Received 18 August 1993

W.K. Nicholson was supported by NSERC Grant A8075 and by The Ohio State University.

Copyright Clearance Centre, Inc. Serial-fee code: 0004-9729/94 \$A2.00+0.00. 
It follows from [1, Proposition 5] that $R$ is left Artinian. To show that $R$ is left serial, we prove the following statement by induction on the index of nilpotency of $J=J(R)$ :

If $1=e_{1}+\cdots+c_{n}$ in $R$ where the $e_{i}$ are orthogonal

local idempotents, then $R e_{i}$ is uniserial for each $i$.

If $J=0$ this is clear because $R$ is semisimple. In general, let $1=e_{1}+\cdots+e_{n}$ as in $\left({ }^{*}\right)$. Writing $S=\operatorname{Soc}\left({ }_{R} R\right)$, we have $S e_{i}=\operatorname{Soc} R e_{i}$ and this is simple and essential in $R e_{i}$ for each $i$ by [8, Theorem 2.3]. Moreover $\bar{R}=R / S$ inherits our hypotheses and, writing $\bar{r}=r+S$ for $r \in S$, we have $\overline{1}=\bar{e}_{1}+\cdots+\bar{e}_{m}$ for some $m \leqslant n$, and $\bar{R} \bar{e}_{1} \cong R e_{i} / S e_{i}$ for each $i$. It follows by induction that $R e_{i} / S e_{i}$ is uniserial for each $i$, so $R e_{i}$ is uniserial, as required.

The converse to Theorem 1 is not true. Indeed, if $F$ is a field the ring $R=\left[\begin{array}{cc}F & F \\ 0 & F\end{array}\right]$ is Artinian (meaning right and left Artinian) and serial, but is neither right nor left $P$ injective. However we get a converse for local rings.

The following result will be needed and extends the result (see Faith [3], p.133) that a commutative valuation ring is $P$-injective if and only if every element is a zero divisor or a unit. Let $Z\left(R_{R}\right)$ denote the right singular ideal of a ring $R$.

LEMMA 1. The following are equivalent for a left uniserial ring $R$.

(1) $R$ is right $P$-injective.

(2) $J(R)=Z\left(R_{R}\right)$

(3) If $a \in R$, then either $R a=R$ or $r(a)$ is essential in $R_{R}$.

Proof: (1) $\Rightarrow$ (2) This follows by $[8$, Theorem 2.1].

$(2) \Rightarrow(3)$ By hypotheses $J=J(R)$ is the unique maximal left ideal of $R$. Hence $R a \neq R$ implies $a \in Z\left(R_{R}\right)$ by (2).

(3) $\Rightarrow$ (1) If $b \in \ell(r(a))-R a$ then $R a \subseteq R b$ by hypothesis, say $a=c b$. Also, $r(b) \supseteq r[\ell(r(a))]=r(a)$, whence $b R \cap r(c)=0$. Thus $r(c)$ is not essential so $R c=R$ by (3). This implies that $b \in R a$, a contradiction.

THEOREM 2. Let $R$ be a left uniserial, right perfect ring. Then:

(1) $R$ is left Artinian and right $C P$-injective.

(2) $R$ is left self-injective if and only if $b R=R b$ for all $b \in R$.

ProOF: Write $J=J(R)$ so that $R=J^{0} \supset J \supset J^{2} \supset \cdots \supset J^{n-1} \supset J^{n} \supset \cdots$ is a composition series of $R$. Let $x_{i} \in J^{i}-J^{i+1}$, then $J^{i}=R x_{i}$. Now $R x_{i+1}=J^{i+1}=$ $J J^{i}=J R x_{i}=J x_{i}$. Thus $x_{i+1}=t_{i} x_{i}$, for some $t_{i} \in J$. By left $T$-nilpotency of $J$, $J^{n}=0$ for some $n$ and so $R$ is semiprimary.

Claim 1. If $L$ is a left ideal of $R$, then $L=J^{m}$ for some $m=0,1, \cdots, n$. 
Proof: If $L \neq 0$, let $L \subseteq J^{m}, L \nsubseteq J^{m+1}$. Then $J^{m+1} \subset L \subseteq J^{m}$ because ${ }_{R} R$ is uniserial so $L=J^{m}$ because $J^{m} / J^{m+1}$ is simple.

Claim 2. $r\left(J^{m}\right)=J^{n-m}=\ell\left(J^{m}\right)$ for all $m=0,1,2, \ldots, n$.

Proof: $J^{n-m} \subseteq r\left(J^{m}\right)$ so $r\left(J^{m}\right)=J^{t}$ where $t \leqslant n-m$. But then $0=J^{m+t}$ so $m+t \geqslant n$. Hence $t=n-m$. Similarly $\ell\left(J^{m}\right)=J^{n-m}$.

(1) $R$ is left Artinian by Claim 1 . Since our hypotheses are inherited by images, it remains to show that $R$ is right $P$-injective. By Lemma 1, it suffices to show that $Z\left(R_{R}\right)=J$. But if $a \in J$ we have $J^{n-1} \subseteq r(a)$, so it suffices to show that $J^{n-1}=$ $\operatorname{Soc}\left(R_{R}\right)$ ( $\operatorname{Soc}\left(R_{R}\right)$ is right essential because $R$ is semiprimary). Let $\operatorname{Soc}\left(R_{R}\right)=J^{m}$. Then $J^{m+1}=\operatorname{Soc}\left(R_{R}\right) J=0$, so $m \geqslant n-1$; as $\operatorname{Soc}\left(R_{R}\right) \neq 0$, we have $m=n-1$, as required.

(2) Since $R$ is a left principal ideal ring (Claim 1), we show that $R$ is left $P$ injective; equivalently that $r(\ell(b))=b R$ for all $b \in R$. Write $R b=J^{m}$ and $\ell(b)=J^{t}$. Then $J^{t+m}=J^{t} R b=0$ so $t+m \geqslant n$. On the other hand $J^{n-m} b \subseteq J^{n-m} J^{m}=0$, so $J^{n-m} \subseteq J^{t}$, whence $n-m \geqslant t$. It follows that $t+m=n$, so $r[\ell(b)]=r\left(J^{n-m}\right)=$ $J^{n-(n-m)}=J^{m}=R b$ by Claim 2. Now (2) follows.

If $R$ is assumed to be both left and right $P$-injective in Theorem 1, we obtain a much stronger conclusion.

THEOREM 3 . The following are equivalent for a ring $R$ :

(1) $R$ is left perfect and both right and left $C P$-injective.

(2) $R$ is completely quasi-Frobenius.

Proof: Since $(2) \Rightarrow(1)$ is clear, assume (1). The hypotheses hold in any image of $R$, so it suffices to show that $R$ is quasi-Frobenius. Theorem 1 implies that $R$ is Artinian and serial. Moreover $Z\left(R_{R}\right)=J(R)=Z\left({ }_{R} R\right)$ by [8, Theorem 2.1]. Now $\ell(J)=$ $\operatorname{Soc}\left(R_{R}\right)$ because $R$ is semiprimary, whence $\ell\left(Z\left({ }_{R} R\right)\right)=\operatorname{Soc}\left(R_{R}\right)$. But $\operatorname{Soc}\left({ }_{R} R\right)$. $Z\left({ }_{R} R\right)=0$ always holds, and it follows that $\operatorname{Soc}\left({ }_{R} R\right) \subseteq \operatorname{Soc}\left(R_{R}\right)$. The other inclusion is similar, so $\operatorname{Soc}\left({ }_{R} R\right)=\operatorname{Soc}\left(R_{R}\right)$. Now let $1=e_{1}+\cdots+e_{n}$ where the $e_{i}$ are orthogonal local idempotents. Since $R$ is left serial, it follows that $\operatorname{Soc}\left(R e_{i}\right)$ is simple for each $i$. Similarly $\operatorname{Soc}\left(e_{i} R\right)$ is simple for each $i$. Since $R$ is (two-sided) Artinian and $\operatorname{Soc}\left({ }_{R} R\right)=\operatorname{Soc}\left(R_{R}\right)$, this implies that $R$ is quasi-Frobenius by [6, p.342].

A ring $R$ is called right 2-injective if $R$-maps $T \rightarrow R$ can be extended to $R$ for all 2-generated right ideals $T$ of $R$. Then [8, Corollary 2.5] implies that a left perfect right 2 -injective ring is left $P$-injective. Hence Theorem 3 gives:

THEOREM 4. The following are equivalent for a ring $R$ :

(1) $R$ is left perfect and completely right 2 -injective.

(2) $R$ is completely quasi-Frobenius. 
If $R$ is commutative, the hypotheses in (1) of Theorem (3) can be relaxed. A commutative ring $R$ is called min-injective if, for each minimal ideal $K$ of $R$, each $R$-linear map $K \rightarrow R$ is multiplication by an element of $R$ (equivalently $\operatorname{ann}^{2} K=K$ where $\left.\operatorname{ann}^{2} K=\operatorname{ann}(\operatorname{ann} K)\right) . \quad R$ is called a min-CS ring if each (minimal) ideal is essential in a direct summand of $R$. Note that $Z$ the ring of integers is completely min-injective and completely min-cs, but it is not $P$-injective.

The following Lemma will be needed.

Lemma 2. Every commutative, semiprime $P$-injective ring $R$ is (von Neumann) regular.

ProOF: Given $a \in R$ we have ann $\left(a^{2}\right) \subseteq$ ann $(a)$ because $R$ is semiprime. Hence $\sigma: a^{2} R \rightarrow a R$ is well-defined by $\sigma\left(a^{2} r\right)=a r$. Since $R$ is $P$-injective, $\sigma=b$. is multiplication by $b \in R$. Hence $a=\sigma\left(a^{2}\right)=b a^{2}=a b a$.

THEOREM 5. The following are equivalent for a commutative ring $R$ :

(1) $R$ is completely quasi-Frobenius.

(2) $R$ is perfect and completely min-injective.

(3) $R$ is perfect and is a completely min-cs ring.

(4) $R$ has Krull dimension and is completely $P$-injective.

(5) $R$ is completely $G P F$-ring.

Proof: Clearly, (1) implies each of (2), (3), (4) and (5).

$(2) \Rightarrow(1)$ It is routine to verify that a finite product of commutative rings is mininjective if and only if each factor is min-injective. Hence we may assume that $R$ is local. Moreover (2) is inherited by ring images, so it suffices to show that $R$ is quasiFrobenius. Now $S=\operatorname{Soc}(R)$ is essential in $\mathrm{R}$ ( $R$ is perfect) and $S$ is homogeneous (two isomorphic simple ideals are equal because $R$ is min-injective). It follows that $S$ is simple, so $R$ is uniform. Furthermore, each non-zero $R$-module has a maximal submodule ( $R$ is perfect) so $R$ is Noetherian by a theorem of Shock [9]. As $J(R)$ is nil, this implies that $R$ is semiprimary, hence Artinian. Now $R$ is quasi-Frobenius by $[6$, p.342].

(3) $\Rightarrow(1)$. As (3) is inherited by ring images, we show that $R$ is quasi-Frobenius. Write $S=\operatorname{Soc}(R)$ and $J=J(R)$. We have $S=\operatorname{ann} J$ (as $R / J$ is semisimple) and it follows that $\operatorname{ann}^{2} S=S$. This gives:

Claim 1. $\operatorname{ann}^{2} K=K$ for all simple ideals $K$.

Proof: First $K \subseteq S$ so $\operatorname{ann}^{2} K \subseteq \operatorname{ann}^{2} S=S$, whence $\operatorname{ann}^{2} K$ is semisimple. Since $K \subseteq \operatorname{ann}^{2} K$, it suffices to show that $K \subseteq \mathrm{ann}^{2} K$ is an essential extension. But $K \subseteq R e$ is essential for some $e^{2}=e \in R$, so $K \subseteq \operatorname{ann}^{2} K \subseteq \operatorname{ann}^{2} R e=R e$.

The claim shows that $R$ is min-injective, so (2) $\Rightarrow(1)$ completes the proof. 
(4) $\Rightarrow(1)$. As (4) is inherited by images, we show that $R$ is quasi-Frobenius.

Claim 2. Every prime ideal of $R$ is maximal.

Proof: $R / P$ is regular by Lemma 2 , and so is semisimple (it has Krull-dimension and so is finite dimensional). Since $P$ is prime, $R / P$ is simple.

Writing $J=J(R)$, it follows from Claim 2 that $J$ is nil and so is nilpotent (see [4]). Furthermore, $R / J$ is regular (by Lemma 2) and finite dimensional (it has Krull dimension), and so is semisimple. Thus $R$ is semiprimary and we are done by (2) $\Rightarrow$ (1).

(5) $\Rightarrow(2)$. By $[8$, Theorem 2.3] and [1, Proposition 5], $R$ is Artinian. Since $G P F$-rings are $P$-injective we are done.

Remarks. (i) It is easy to see that every regular ring is a left and right $C P$-injective ring and by Theorem 3 the converse is not true. In fact $Z_{q^{2}}$, where $q$ is a prime number, is a commutative $C P$-injective ring which is not regular.

(ii) In [2, Remark 2 on p.36] Camillo has an example of a commutative, semiprimary, local $P$-injective ring which is not injective.

(iii) See Faith [3, Proposition 25.4.6B, p.238] for a complete description of the class of completely $Q F$-rings. They are precisely the Artinian principal ideal rings.

(iv) The ring $R=\left[\begin{array}{cc}F & F \\ 0 & F\end{array}\right]$, where $F$ is a field, is an Artinian completely $C S$-ring which is not right min-injective. However, every proper homomorphic image of $R$ is injective.

(v) In general a module ${ }_{R} M$ is called a $(\min ) C S$-module if every (simple) submodule of $M$ is essential in a summand of $M$. ( $\min ) C S$-modules are called (simple)extending modules by Harada [5]. For a full account of $C S$-modules see Mohamed and Müller [7].

\section{References}

[1] J.A. Beachy, 'On quasi-artinian rings', J. London Math. Soc. 3 (1971), 449-452.

[2] V. Camillo, 'Commutative rings whose principal ideals are annihilators', Portugal. Math. 46 (1989), 33-37.

[3] C. Faith, Algebra II, Ring theory (Springer-Verlag, Berlin, Heidelberg, New York, 1976).

[4] R. Gordon and J.C. Robson, 'Krull dimension', Mem. Amer. Math. Soc. 133 (1973).

[5] M. Harada, 'On modules with extending properties', Osaka J. Math. 19 (1982), 203-215.

[6] F. Kasch, Modules and rings, London. Math. Soc. Monographs 17 (Academic Press, New York, 1982).

[7] S.H. Mohamed and B.J. Müller, Continuous and discrete modules, London Math. Soc. Lecture Notes Series 147 (Cambridge University Press, Oxford, 1990). 
[8] W.K. Nicholson and M.R. Yousif, 'Principally injective rings', J. Algebra (to appear).

[9] R.C. Shock, 'Dual generalizations of the artinian and neotherian conditions', Pacific J. Math. 54 (1974), 227-235.

Department of Mathematics

University of Calgary

Calgary, Alberta

Canada T2N 1N4
Department of Mathematics

The Ohio State University at Lima

4240 Campus Drive

Lima OH 45804

United States of America 